\title{
Ausschuß der Weiterbildungsinstitute im DVT
}

Auf der letzten Mitgliederversammlung der Institute im DVT in Mannheim am 20.1.94 legten die beiden Sprecher des Institutsausschusses (Prof. Dr. D. Zimmer, Tubingen, und Prof. Dr. Manfred Fichter, München) den Bericht für das erste Jahr des neuen Verbandes vor und wurden von der Versammlung entlastet. Aus formalen Gründen wurde die Wahl des ersten Sprechers vom Vorjahr wiederholt. Einstimmig wurde Dirk Zimmer, der bisherige erste Sprecher, für zwei weitere Jahre gewählt.

Die nächste Jahrestagung des DVT wird vom 20. bis 22.1.1995 in Berlin vom dortigen Institut IVB-Berlin ausgerichtet.

Die bisherige Politik der Sprecher, insbesondere in der politischen Arbeit um das Psychotherapeutengesetz, wurde begrüßt und bestätigt. Versuche einer Ärztegruppe, über eine Stellungnahme bei der Anhörung des Gesundheitsausschusses im November 1993 die gleichberechtigte Konzeption des Berufes Psychologischer Psychotherapeuten zu verhindern, wurden sehr deutlich zurückgewiesen (ebenso auf der MV des DVT am folgenden Tag).

Der Sprecher der Institute nahm an mehreren Sitzungen des Gesprächskreises Psychotherapeutengesetz (ehemals Spiegel-AG) teil sowie an verschiedenen Kontakten zu Politikern. Angesichts der Tatsache, daß zum Zeitpunkt der Veröffentlichung dieses Berichtes aktuellere Informationen zum Stand des Psychotherapeutengesetzes vorliegen werden, sollen hierzu keine weiteren Details dargestellt werden.

Interna des Ausschusses der Weiterbildungsinstitute

Das bisherige Institut IVT-Bad Dürrheim hat die Arbeit organisatorisch und personell auf eine neue, breitere Basis gestellt und firmiert ab sofort als IVT-Freiburg im Institutsausschuß. Als Vollmitglied aufgenommen wurde das AGVT-Köln und das IVV-Berus, als assoziiertes Mitglied des TIV-Thüringen.

Entsprechend dem Rahmenthema des letzten Kongresses des DVT im Januar 1994 am Zentralinstitut für seelische Gesundheit in Mannheim wird im Rahmen des Weiterbildungsausschusses intensiv über Richtlinien und Maßnahmen zur Qualitätssicherung verhaltenstherapeutischer Weiterbildungsgänge in den Instituten diskutiert.

Ausschuß der Weiterbildungsinstitute im DVT

Geschäftsstelle:

Waldhäuserstraße 48

D-72076 Tubingen

Tel.(. (07071)610838

Fax (07071) 760243 